\title{
LEPTOSPIROSIS INFANTIL EN PACIENTES CON SÍNDROME FEBRIL EN LA REGIÓN DE URABÁ, COLOMBIA
}

\author{
Janeth Pérez-García ${ }^{1, a}$, Margarita Arboleda ${ }^{2, b}$, Piedad Agudelo-Flórez $3, c$
}

\begin{abstract}
RESUMEN
El objetivo del estudio fue describir los casos de leptospirosis infantil en cuatro municipios de la región de Urabá (Colombia) y factores relacionados con severidad de los cuadros clínicos. Se realizó un estudio transversal analítico retrospectivo de 74 pacientes menores de 17 años con síndrome febril por leptospirosis entre 2010 y 2012 . El mayor número de casos correspondió a niños de áreas urbanas (57/74; 77\%), entre 10 y 14 años (33/74; 44,5\%). La respuesta por MAT más frecuente fue a los serogrupos Grippothyposa y Bratislava, asociados a reservorios silvestres y pecuarios respectivamente. Se encontró asociación entre la presencia de animales domésticos dentro de la vivienda (OR 2,73; IC95\% 0,98-7,60; $p=0,05$ ) y la severidad de la enfermedad. El riesgo de leptospirosis infantil severa se relaciona con la tenencia de animales domésticos dentro de la vivienda.
\end{abstract}

Palabras clave: Leptospira, Weil, pediatría, epidemiología, zoonosis

\section{CHILDHOOD LEPTOSPIROSIS IN PATIENTS WITH FEBRILE SYNDROME IN THE REGION OF URABÁ, COLOMBIA}

\begin{abstract}
The aim of the study was to discover cases of childhood leptospirosis in four municipalities in the region of Urabá (Colombia) and the factors related to the severity of clinical manifestations. A retrospective cross-sectional study assessed 74 children aged younger than 17 years with febrile syndrome due to leptospirosis between 2010 and 2012 . The majority of cases were in children from urban areas $(57 / 74 ; 77 \%)$, between 10 and 14 years of age $(33 / 74 ; 44.5 \%)$. The microscopic agglutination test (MAT) revealed that the most frequent serogroups were Grippotyphosa and Bratislava, which are associated with wildlife and livestock reservoirs, respectively. There was a association between the presence of household pets and the severity of the disease (odds ratio $[\mathrm{OR}] 2.73$; $95 \%$ confidence interval $[\mathrm{Cl}]=0.98-7.60$; $\mathrm{p}=0.05)$. The risk of severe childhood leptospirosis is linked to having household pets.
\end{abstract}

Key words: Leptospira, Weil, pediatrics, epidemiology, zoonoses

\section{INTRODUCCIÓN}

La leptospirosis es considerada una enfermedad reemergente en todo el mundo, no solo por el aumento de casos, sino también por las diferentes presentaciones clínicas y el aumento de hospederos y serovares de la bacteria circulando, incluso, en ambientes no convencionales ${ }^{(1,2)}$. A nivel mundial se registran aproximadamente 1,03 millones de casos cada año y en Latinoamérica se estiman hasta 36 mil casos anuales ${ }^{(3)}$; en Colombia se reportan prevalencias desde el $6 \%$ en el departamento del Tolima hasta el $82,7 \%$ en la zona cafetera, y en Antioquia se reporta una prevalencia del $35,6 \%{ }^{(4)}$. De acuerdo con las cifras oficiales epidemiológicas, la región de Urabá registró el
$58,1 \%$ de los casos reportados en el departamento de Antioquia, durante el año $2014{ }^{(5)}$.

El conocimiento de la historia natural de la enfermedad y de los factores de riesgo, se basa en el estudio de casos presentados en adultos, sin embargo, en áreas endémicas se presentan igualmente casos pediátricos, y es importante explorar las características clínicas y la forma de presentación de la leptospirosis en este grupo de edad, ya que existe subregistro y subdiagnóstico ${ }^{(6)}$.

Es así como la tasa de incidencia en la población pediátrica es baja, posiblemente, como ya se estipuló, debido al subdiagnóstico, por ser la presentación clínica similar a otras enfermedades infecciosas en la

\footnotetext{
Universidad CES. Medellín, Colombia.

Instituto Colombiano de Medicina Tropical. Apartadó, Colombia.

Facultad de Medicina, Universidad CES. Medellín, Colombia.

a Doctorado Epidemiología y Bioestadística. ${ }^{\mathrm{b}}$ MD. Ms Medicina Tropical. ${ }^{\mathrm{c}}$ Bióloga. PhD Ciencias Biomédicas.

Recibido: 12/01/2016 Aprobado: 12/10/2016
} 
mayoría de países tropicales, como la fiebre tifoidea, malaria y brucelosis ${ }^{(7)}$ y por la falta de documentación del diagnóstico dirigido de esta enfermedad. En un estudio realizado en Tanzania se reportó un 13\% de niños positivos para Leptospira spp. mediante pruebas serológicas (microaglutinación y ensayo inmunoenzimático), entre 370 pacientes atendidos con síndrome febril ${ }^{(6)}$. Los signos clínicos, generalmente, son moderados y la mortalidad oscila entre 1,9 y $13,8 \%$ en niños hospitalizados.

Incluso, se sugiere en la literatura que a pesar de que los niños estén expuestos a la bacteria, podrían no padecer signos clínicos asociados ${ }^{(8)}$. El propósito de este trabajo fue determinar características de la leptospirosis infantil y factores asociados, con severidad en la presentación clínica, en una región endémica para esta enfermedad.

\section{MATERIALES Y MÉTODOS}

Estudio transversal analítico con base en información secundaria, obtenida de un proyecto macro sobre leptospirosis. Este estudio revisó la información clínica y sociodemográfica de 479 pacientes con síndrome febril que ingresaron a instituciones de salud en cuatro municipios de la región de Urabá (Apartadó, Carepa, Chigorodó y Turbo), entre los años 2010 y 2012, obteniéndose información de 74 pacientes pediátricos con edades entre 5 y 17 años.

El uso de la información contenida en la base de datos del proyecto macro fue aprobado por el Comité de Ética de la Universidad CES (Cod. 309, Acta 70 de 2014).

El diagnóstico de leptospirosis se documentó mediante hemocultivo positivo, seroconversión, alza de títulos por microaglutinación (MAT), o prueba de inmunofluorescencia indirecta (IFI) en fase convaleciente, pruebas que se realizan en el Instituto Colombiano de Medicina Tropical. El hemocultivo se realizó en medio semisólido de Fletcher, y la prueba IFI se estandarizó en el ICMT por Agudelo-Flórez et al (9), considerando títulos positivos de $\lg M \geq 1: 40$ y de $\lg G$ $\geq 1: 80$.

Los anticuerpos por MAT, reconocida como la prueba de referencia por la Organización Mundial de la Salud, se consideraron positivos con un alza cuádruple de títulos en muestra convaleciente y un título $\geq 1: 400$ en el caso de disponerse de una sola muestra. Como se aprecia en la Figura 1, se recategorizó esta variable según la fuente teórica de origen doméstico o silvestre de acuerdo con la respuesta serológica al serogrupo identificado en cada uno de los pacientes.

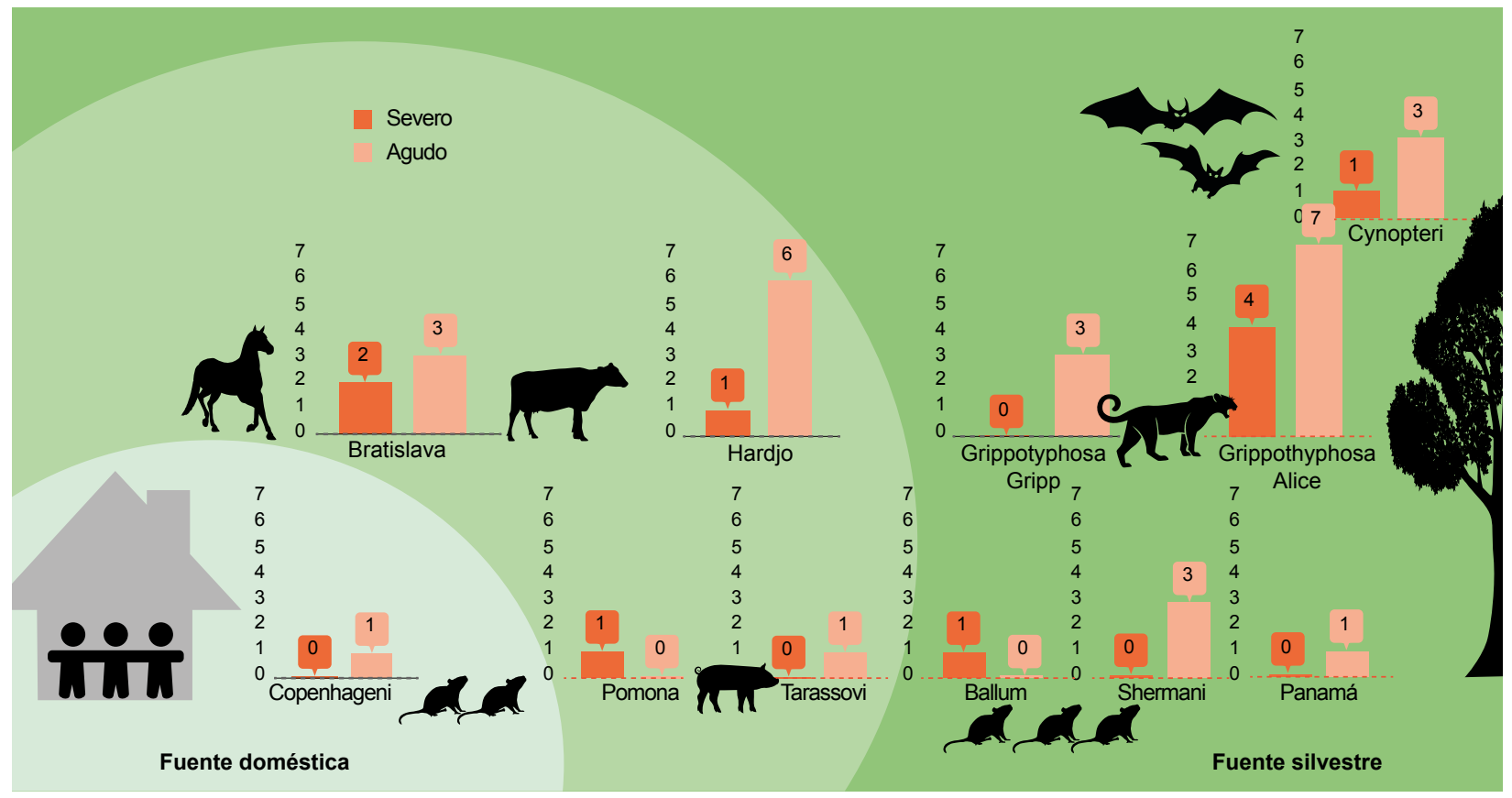

Figura 1. Distribución de los serogrupos obtenidos por MAT de acuerdo con la severidad de la presentación y la fuente teórica de origen animal ${ }^{7-10}$. Se incluyen solo los pacientes positivos a MAT y con serogrupo único, excluyendo resultados con coaglutinación de más de dos grupos 
La presentación de la enfermedad se determinó teniendo en cuenta caso severo, aquel paciente que necesitara hospitalización con al menos compromiso de uno o más órganos vitales, o que hubiese requerido soporte ventilatorio, ingreso a una unidad de cuidados intensivos o que hubiese fallecido. Se consideró caso leve al paciente que presentó fiebre, cefalea, mialgias y otros síntomas generales, manejado ambulatoriamente. No se incluyó categoría moderado porque esta no es considerada por la Organización Mundial de la Salud (10).

Se determinó como variable dependiente la presentación clínica severa de la enfermedad. Se usó la prueba chi cuadrado para identificar asociación entre las variables de riesgo cualitativas. Los análisis fueron realizados en el software SPSS 21.0 (licencia Universidad CES), utilizando un nivel de confianza del $95 \%$ y un valor $p<0,05$ para la significancia estadística. Las figuras fueron realizadas en la página web Piktochart (distribución libre).

\section{RESULTADOS}

El 73\% (54/74) de los pacientes pediátricos con síndrome febril por leptospirosis en los tres años de estudio fueron del sexo masculino, con una edad promedio de $12( \pm 3,5)$ años, siendo las pertenencias étnicas predominantes mestizo y blanco con 47,3 y $35,1 \%$ respectivamente. En la Tabla 1 se presenta la distribución de las variables sociodemográficas según grupo etario en los niños con leptospirosis. Un 29,7\% (22/74) de los pacientes pediátricos se clasificó como caso severo y el 70,3\% (52/74) restante como caso leve. Cinco pacientes con enfermedad severa tenían entre 5 y 9 años $(22,7 \%)$, 10 entre 10 y 14 años (45,5\%) y 7 entre 15 y 17 años $(31,8 \%)$, mientras que la distribución de enfermedad leve fue de $15(28,8 \%), 23(44,2 \%)$ y $14(27 \%)$ niños, dentro de estos grupos de edad, respectivamente. Se registraron dos casos fatales.

La figura 1 describe el número de casos por serogrupo de acuerdo con la clasificación de severidad, siendo más prevalente la respuesta de anticuerpos contra Leptospira santarosai serogrupo Grippotyphosa serovar Alice $(25 \%)$ y Leptospira interrogans serogrupo Hardjo (16\%). Los pacientes positivos a serogrupos de origen silvestre tuvieron títulos superiores a 1:800, mientras que los títulos contra los serogrupos de origen pecuario fueron menores a 1:400.

La Tabla 2 describe los signos clínicos presentes en la población de estudio, destacándose una mayor proporción de ictericia $(45,5 \%)$, dolor abdominal y manifestaciones hemorrágicas $(40,9 \%)$, hepatomegalia $(31,8 \%)$, y deshidratación $(27,3 \%)$ en los casos severos. El $22,7 \%$ de los pacientes tuvo prueba del torniquete positiva. Esta tabla incluye asociación significativa entre la presencia del signo y la presentación del cuadro clínico. Las variables clínicas de deshidratación,

Tabla 1. Distribución de las variables sociodemográficas según grupo etario. Leptospirosis infantil, Urabá, Colombia

\begin{tabular}{|c|c|c|c|c|c|c|c|c|}
\hline & \multicolumn{6}{|c|}{ Grupo etario } & & \\
\hline & \multicolumn{2}{|c|}{ 05-09 años } & \multicolumn{2}{|c|}{ 10-14 años } & \multicolumn{2}{|c|}{ 15-17 años } & \multicolumn{2}{|c|}{ Total } \\
\hline & $\mathbf{n}$ & $(\%)$ & $\mathrm{n}$ & (\%) & $\mathbf{n}$ & $(\%)$ & $\mathbf{n}$ & (\%) \\
\hline \multicolumn{9}{|l|}{ Sexo } \\
\hline Masculino & 15 & $(75,0)$ & 25 & $(75,8)$ & 14 & $(66,7)$ & 54 & $(73,0)$ \\
\hline Femenino & 5 & $(25,0)$ & 8 & $(24,2)$ & 7 & $(33,3)$ & 20 & $(27,0)$ \\
\hline \multicolumn{9}{|l|}{ Escolaridad } \\
\hline No estudiante & 9 & $(45,0)$ & 11 & $(33,3)$ & 3 & $(14,3)$ & 23 & $(31,1)$ \\
\hline Estudiante & 11 & $(55,0)$ & 21 & $(63,6)$ & 18 & $(85,7)$ & 50 & $(67,6)$ \\
\hline No evaluado & 0 & $(0,0)$ & 1 & $(3,0)$ & 0 & $(0,0)$ & 1 & $(1,4)$ \\
\hline \multicolumn{9}{|l|}{ Municipio } \\
\hline Apartadó & 13 & $(65,0)$ & 19 & $(57,6)$ & 11 & $(52,4)$ & 43 & $(58,1)$ \\
\hline Carepa & 2 & $(10,0)$ & 7 & $(21,2)$ & 5 & $(23,8)$ & 14 & $(18,9)$ \\
\hline Chigorodó & 1 & $(5,0)$ & 2 & $(6,1)$ & 0 & $(0,0)$ & 3 & $(4,1)$ \\
\hline Turbo & 4 & $(20,0)$ & 5 & $(15,2)$ & 5 & $(23,8)$ & 14 & $(18,9)$ \\
\hline \multicolumn{9}{|c|}{ Ubicación de la vivienda } \\
\hline Urbana & 15 & $(75,0)$ & 27 & $(81,8)$ & 15 & $71,4)$ & 57 & $(77,0)$ \\
\hline Rural & 5 & $(25,0)$ & 6 & $(18,2)$ & 6 & $28,6)$ & 17 & $(23,0)$ \\
\hline Total & 20 & $(100,0)$ & 33 & $(100,0)$ & 21 & $(100,0)$ & 74 & $(100,0)$ \\
\hline
\end{tabular}


Tabla 2. Asociación entre la presencia de signos clínicos evaluados al momento de la consulta y su categoría de severidad

\begin{tabular}{|c|c|c|c|c|}
\hline & \multirow{2}{*}{$\begin{array}{c}\text { Sin leptospirosis } \\
\mathbf{n}(\%)\end{array}$} & \multicolumn{2}{|c|}{ Con leptospirosis } & \multirow[b]{2}{*}{ Valor-p } \\
\hline & & Severo n (\%) & Leve n (\%) & \\
\hline Deshidratación & $63(85,1)$ & $6(27,3)$ & $1(1,9)$ & 0,001 \\
\hline Diaforesis & $67(90,5)$ & $2(9,1)$ & $0(0)$ & 0,003 \\
\hline Mialgia & $53(71,6)$ & $4(18,2)$ & $14(26,9)$ & 0,331 \\
\hline Ictericia & $59(79,7)$ & $10(45,5)$ & $5(9,6)$ & 0,000 \\
\hline Cianosis & $67(90,5)$ & $1(4,5)$ & $0(0)$ & 0,003 \\
\hline Murmullo vesicular disminuido & $70(94,6)$ & $0(0)$ & $2(3,8)$ & 0,537 \\
\hline Ruidos sobreagregados pulmonares & $69(93,2)$ & $1(4,5)$ & $3(5,8)$ & 0,786 \\
\hline Dolor abdominal & $43(58,1)$ & $9(40,9)$ & $22(42,3)$ & 0,911 \\
\hline Hepatomegalia & $59(79,7)$ & $7(31,8)$ & $5(9,6)$ & 0,016 \\
\hline Esplenomegalia & $70(94,6)$ & $1(4,5)$ & $0(0)$ & 0,103 \\
\hline Adenomegalias & $59(79,7)$ & $2(9,1)$ & $13(25)$ & 0,120 \\
\hline Brotes cutáneos & $57(77)$ & $2(9,1)$ & $6(11,5)$ & 0,932 \\
\hline Manifestaciones hemorrágicas & $46(62,2)$ & $9(40,9)$ & $19(36,5)$ & 0,723 \\
\hline Prueba del torniquete positiva & $50(67,6)$ & $5(22,7)$ & $9(17,3)$ & 0,181 \\
\hline Petequias & $62(83,8)$ & $2(9,1)$ & $2(3,8)$ & 0,055 \\
\hline
\end{tabular}

ictericia, hepatomegalia y petequias presentaron valores p por debajo de 0,05.

Se exploró asociación entre algunos factores de riesgo, como zona de ubicación de vivienda, presencia de roedores y animales silvestres dentro de la casa, antecedentes de inundación en el lugar donde vive, con la severidad del cuadro clínico del paciente, y no se encontró diferencia significativa como se presenta en la Tabla 3. Solo se presentó asociación de los casos severos de la enfermedad con la presencia de animales domésticos en la vivienda (IC 95\%: 0,98-7,60; $p=0,05$ ).

Tabla 3. Análisis bivariado de los factores de riesgo según presentación clínica de la leptospirosis infantil en la región de Urabá, Colombia

\begin{tabular}{|c|c|c|c|c|c|c|}
\hline & \multicolumn{2}{|c|}{ Severo } & \multicolumn{2}{|c|}{ Leve } & \multirow{2}{*}{$\begin{array}{c}\text { OR crudo } \\
\text { (IC } 95 \%)\end{array}$} & \multirow[b]{2}{*}{ valor $p$} \\
\hline & $\mathbf{n}$ & $\%$ & $\mathbf{n}$ & $\%$ & & \\
\hline \multicolumn{7}{|c|}{ Zona de ubicación de la vivienda } \\
\hline Rural & 6 & 27,3 & 11 & 21,2 & 1,00 & \\
\hline Urbana & 16 & 72,7 & 41 & 78,8 & $1,40(0,44-4,44)$ & 0,5674 \\
\hline \multicolumn{7}{|c|}{ Asistencia a institución educativa } \\
\hline No & 10 & 45,5 & 14 & 26,9 & 1,00 & \\
\hline Sí & 12 & 54,5 & 38 & 73,1 & $2,26(0,80-6,39)$ & 0,1196 \\
\hline \multicolumn{7}{|c|}{ Antecedentes inundación en vivienda } \\
\hline Sí & 13 & 59,1 & 23 & 44,2 & 1,00 & \\
\hline No & 9 & 40,9 & 29 & 55,8 & $1,82(0,66-5,01)$ & 0,2424 \\
\hline \multicolumn{7}{|c|}{ Origen de la Leptospira spp aislada } \\
\hline Doméstico y pecuario & 16 & 72,7 & 35 & 67,3 & 1,00 & \\
\hline Silvestre & 6 & 27,3 & 17 & 32,7 & $1,30(0,43-3,90)$ & 0,6452 \\
\hline \multicolumn{7}{|c|}{ Presencia de animales domésticos en la vivienda } \\
\hline Presencia & 9 & 40,9 & 34 & 65,4 & $2,73(0,98-7,60)$ & 0,0511 \\
\hline Ausencia & 13 & 59,1 & 18 & 34,6 & 1,00 & \\
\hline Total & 22 & 100 & 52 & 100 & & \\
\hline
\end{tabular}




\section{DISCUSIÓN}

La frecuencia de la enfermedad en el presente estudio fue de $58,7 \%$ (74 casos positivos/ 126 niños incluidos en el estudio) hallazgo considerablemente alto, si se compara con el $13 \%$ referido recientemente en niños entre 2 y 13 años de Tanzania ${ }^{(6)}$. Aunque se ha considerado, clásicamente, que el mayor número de casos se presenta en zonas rurales y de tipo ocupacional ${ }^{(11)}$, en el presente estudio hubo una mayor proporción de casos en el área urbana, hecho probablemente explicable por la distribución de la población en estos municipios y también por las condiciones socioambientales ${ }^{(12,13)}$. En este sentido, es posible mencionar que los hallazgos del presente estudio son parcialmente similares a los propuestos por Mwachui en su revisión sistemática de la enfermedad ${ }^{(11)}$, resaltando la leptospirosis urbana como más virulenta, de acuerdo con el modelo aquí planteado, que relaciona la procedencia urbana con la severidad de la enfermedad.

Suarez Hernández en su investigación sobre leptospirosis reporta un mayor número de casos en niños entre 10 y 14 años ${ }^{(14)}$, dato concordante con los hallazgos del presente estudio. Este grupo de edad coincide con la escolarización de la población infantil y su asistencia a instituciones educativas, en donde podrían presentarse otros comportamientos de riesgo que no fueron evaluados directamente por la encuesta.

Hubo un mayor número de casos leves $(70,3 \%)$ y una baja letalidad $(2,7 \%)$ en la población infantil que enfermó de leptospirosis en el presente estudio, hallazgos que coinciden con un reporte que refiere un mayor número de casos pediátricos con formas clínicas leves y baja mortalidad ${ }^{(3)}$. De igual modo, en este grupo poblacional prevalecieron ictericia, hepatomegalia y manifestaciones hemorrágicas en los casos severos. De estos signos y síntomas, la ictericia y el dolor abdominal han estado asociados con severidad también en adultos ${ }^{(15)}$.

Según la respuesta serológica evaluada mediante MAT, los serogrupos más frecuentes fueron aquellos relacionados con especies silvestres, lo cual, sumado al hallazgo de la especie $L$. santarosai Alice serogrupo Grippothyposa, con títulos altos de anticuerpos, podría avalar la hipótesis de la existencia de riesgo de leptospirosis severa en este estudio, por la cercanía del humano con este tipo de animales, hecho que ya ha sido mencionado por otros autores ${ }^{(14,16)}$, incluso referenciado para la zona de Urabá ${ }^{(17)}$. Algunos de estos serovares han sido reconocidos como parte de la dinámica del estudio de las enfermedad de origen no doméstico, incluyendo sus potenciales hospederos y, por ello, fuentes de infección para el hombre ${ }^{(18)}$, entre ellos los murciélagos y marsupiales que pueden encontrarse también en zonas urbanas.

En niños, la presencia de leptospirosis se ha asociado con la tenencia de mascotas ${ }^{(19)}$; en el presente estudio convivir con animales domésticos tuvo influencia significativa en la presentación severa de la leptospirosis. Aunque la información correspondiente a la presencia de animales domésticos no discriminó la especie implicada, es de resaltar que la ausencia del serovar Canicola como serogrupo único detectado por MAT en los sueros de la población infantil sino como coaglutinación, puede ser indicador de que los caninos no son los principales reservorios de la bacteria en la zona urbana de estos municipios del eje bananero de Urabá y, más bien, como se había mencionado previamente, en la dinámica de transmisión en esas áreas urbanas podrían estar involucradas otras especies portadoras, lo mismo que otras interacciones con el ambiente ${ }^{(12)}$.

Los niños pueden estar expuestos a diferentes fuentes de contaminación con Leptospira spp: domiciliarias, peridomiciliarias, en las instituciones educativas o en las actividades recreativas ${ }^{(20)}$, ya que a esta edad el contacto espontáneo con múltiples objetos, superficies, aguas o mascotas, puede tener riesgos inherentes, epidemiológicamente diversos, teniendo en cuenta el comportamiento dinámico de la bacteria en zonas endémicas. Este estudio presenta una frecuencia significativa de la enfermedad en la población infantil y resalta la importancia de las variables ambientales en el conocimiento de la leptospirosis pediátrica, concluyendo que la tenencia de animales domésticos dentro de la vivienda, representa un factor de riesgo para leptospirosis en este grupo poblacional.

Contribución de los autores: JPG participó en análisis estadístico, la interpretación de los datos y redacción del artículo. MAN participó en concepción del estudio, la obtención de financiación, la recolección de los datos y revisión crítica del manuscrito. PAF participo en concepción del estudio, la obtención de financiación, la recolección de los datos y redacción del artículo.

Conflicto de interés: los autores reportan que la presente publicación no tiene ningún conflicto de interés.

Fuente financiación: proyectos financiados por Colciencias Cod. 325649326207-678 y Cod. 122865740423. 


\section{REFERENCIAS BIBLIOGRÁFICAS}

1. Samir A, Soliman R, El-Hariri M, AbdelMoein K, Hatem ME. Leptospirosis in animals and human contacts in Egypt: broad range surveillance. Rev Soc Bras Med Trop. junio de 2015;48(3):272-7.

2. Picardeau M. Leptospirosis: Updating the Global Picture of an Emerging Neglected Disease. Small PLC, editor. PLoS Negl Trop Dis. 24 de septiembre de 2015;9(9):e0004039.

3. Costa F, Hagan JE, Calcagno J, Kane M, Torgerson P, Martinez-Silveira MS, et al. Global Morbidity and Mortality of Leptospirosis: A Systematic Review. Small PLC, editor. PLoS Negl Trop Dis. 17 de septiembre de 2015;9(9):e0003898.

4. Dechner A. A retrospective analysis of the leptospirosis research in Colombia. J Infect Dev Ctries [Internet]. 13 de marzo de 2014 [citado 24 de agosto de 2015];8(3). Disponible en: http:// www.jidc.org/index.php/journal/article/ view/3123

5. Instituto Nacional de Salud. Boletín epidemiológico nacional 2014. Colombia: Instituto Nacional de Salud; (Sivigila 2014).

6. Chipwaza B, Mhamphi GG, Ngatunga SD, Selemani M, Amuri M, Mugasa JP, et al. Prevalence of Bacterial Febrile Illnesses in Children in Kilosa District, Tanzania. Small PLC, editor. PLoS Negl Trop Dis. 8 de mayo de 2015;9(5):e0003750.

7. Thai KTD, Binh TQ, Giao PT, Phuong HL, Hung LQ, Nam NV, et al. Seroepidemiology of leptospirosis in southern Vietnamese children. Trop Med Int Health. mayo de 2006;11(5):738-45.
8. Marotto PC, Marotto MS, Santos DL, Souza TN, Seguro AC. Outcome of leptospirosis in children. Am J Trop Med Hyg. marzo de 1997;56(3):307-10.

9. Agudelo Flórez P, Restrepo M, Lotero MA. Evaluación de la prueba de inmunofluorescencia indirecta para el diagnóstico de leptospirosis humana. Biomédica. 1 de junio de 2006;26(2):216.

10. Organización Mundial de la Salud. Leptospirosis humana: guía para el diagnóstico, vigilancia y control. OPS/ OMS. 2008;12:127.

11. Mwachui MA, Crump L, Hartskeerl R, Zinsstag J, Hattendorf J. Environmental and Behavioural Determinants of Leptospirosis Transmission: A Systematic Review. Small PLC, editor. PLoS Negl Trop Dis. 17 de septiembre de 2015;9(9):e0003843.

12. Agudelo-Flórez P, Restrepo-Jaramillo BN, Arboleda-Naranjo M. Situación de la leptospirosis en el Urabá antioqueño colombiano: estudio seroepidemiológico y factores de riesgo en población general urbana. Cad Saúde Pública. septiembre de 2007;23(9):2094-102.

13. Arroyave E, Londoño AF, Quintero JC, Agudelo-Florez P, Arboleda M, Díaz FJ, et al. Etiología y caracterización epidemiológica del síndrome febril no malárico en tres municipios del Urabá antioqueño, Colombia. Biomédica. 4 de septiembre de 2012;33(0).

14. Suárez Hernández. Brote de leptospirosis en niños con predominio meningoencefálico en el municipio Morón. Rev Cuba Med Trop. 1991;43(2):136-9.
15. Hochedez P, Theodose R, Olive C, Bourhy P, Hurtrel G, Vignier N, et al. Factors Associated with Severe Leptospirosis, Martinique, 20102013. Emerg Infect Dis. diciembre de 2015;21(12):2221-4.

16. Bharti AR, Nally JE, Ricaldi JN, Matthias MA, Diaz MM, Lovett MA, et al. Leptospirosis: a zoonotic disease of global importance. Lancet Infect Dis. diciembre de 2003;3(12):757-71.

17. Yusti D, Arboleda M, Agudelo-Flórez P. Factores de riesgo sociales y ambientales relacionados con casos de leptospirosis de manejo ambulatorio y hospitalario, Turbo-Colombia. Biomédica. 4 de septiembre de 2012;33(Supp 1):117-29.

18. Libraty DH, Myint KSA, Murray CK, Gibbons RV, Mammen MP, Endy TP, et al. A Comparative Study of Leptospirosis and Dengue in Thai Children. Picardeau M, editor. PLoS Negl Trop Dis. 26 de diciembre de 2007;1(3):e111.

19. Wong ML, Kaplan S, Dunkle LM, Stechenberg BW, Feigin RD. Leptospirosis: A childhood disease. J Pediatr. abril de 1977;90(4):532-7.

20. Méndez-Diaz NE, Arada Rodriguez A. Propuesta de estrategia de intervención en salud para la leptospirosis infantil. Rev Cienc Médicas Pinar Rio. 2010;14(1).

Correspondecia: Janeth Pérez-Garcia Dirección: Calle 10A \#22-04. Universidad CES. Facultad de Medicina Veterinaria y Zootecnia. Medellin (Colombia).

Teléfono: (57) (4) 44405555 Extensión 1428-1237. Fax: (57) (4) 2666046.

Correo electrónico:jperez@ces.edu.co

Nuestros artículos se encuentran indizados en: gecpus WwW.scopus.com 